\title{
La influencia de la jurisprudencia de la Corte Internacional de Justicia en la práctica de los tribunales arbitrales del CIADI ${ }^{1}$
}

\author{
The influence of the case law of the International Court of \\ Justice within the practice of ICSID arbitral tribunals
}

\section{Carlos Bellei Tagle}

carlos.bellei@mids.ch

Abogado, Universidad de Chile. LL.M. en Solución de Controversias Internacionales, Universidad de Ginebra e Instituto Universitario de Altos Estudios Internacionales y del Desarrollo, Ginebra. Profesor de la Universidad Alberto Hurtado (Chile).

Resumen: El rol que desempeńa la jurisprudencia de la Corte Internacional de Justicia en el contexto del arbitraje de inversiones ha sido un asunto generalmente subestimado, sobre todo si consideramos que el sistema establecido por el CIADI suele identificarse como un régimen autónomo de solución de controversias. Este artículo adopta una aproximación inversa, y se centra en el análisis de la manera en que los tribunales arbitrales del CIADI han manifestado una significativa apertura hacia esta clase de fallos externos, utilizando con frecuencia las decisiones de la Corte Internacional de Justicia en el proceso de interpretación y aplicación de la normativa específica que rige la solución de disputas sobre inversiones.

Palabras clave: Corte Internacional de Justicia, CIADI, precedente, consistencia.

Abstract: The role played by the case law of the International Court of Justice in the context of investment arbitration has usually been underestimated, especially considering that the system established by the ICSID is often viewed as a self-contained regime of dispute settlement. This paper takes an opposite approach, and focuses on the analysis of the way in which ICSID arbitral tribunals have expressed a significant openness to this kind of external decisions, making use of decisions of the International Court of Justice in the process of interpretation and application of the specific rules governing the settlement of investment disputes.

Keywords: International Court of Justice, ICSID, precedent, consistency.

\footnotetext{
Artículo recibido el 05.08.2014 y aceptado el 14.10.2014.
} 


\section{Introducción}

A menudo, el impacto que tiene la jurisprudencia emanada de la Corte Internacional de Justicia (en adelante «CIJ» o "Corte») más allá de sus fronteras ha sido un asunto subvalorado. No son muchos los trabajos que dan cuenta de la proyección que sus decisiones han tenido en la práctica de otros órganos jurisdiccionales internacionales. A pesar de que el sistema de justicia internacional opera con prescindencia del apego a la regla del precedente y que además carece de una estructura orgánica de tipo jerárquica que sitúe a la CIJ en una posición de superioridad respecto de otros tribunales y cortes, resulta interesante preguntarse por la manera en que diferentes instancias jurisdiccionales de resolución de conflictos han recogido (o desechado) algunos aspectos contenidos en importantes decisiones emanadas del principal órgano judicial de las Naciones Unidas. Lo anterior es especialmente atractivo cuando se explora la influencia de la jurisprudencia de la CIJ en relación con aquellos subsistemas de solución de disputas que se auto conciben como regímenes autónomos ${ }^{2}$, como es el caso de los mecanismos establecidos por la Organización Internacional de Comercio (en adelante «OMC»), la Unión Europea, los tratados de derechos humanos o por las diferentes alternativas para resolver conflictos sobre inversiones extranjeras.

En dicho contexto, uno de los tantos análisis que pueden intentarse consiste en abordar el cómo ciertos aspectos contenidos en sentencias de la CIJ han tenido eco en la labor de numerosos tribunales constituidos al amparo del sistema del Centro Internacional de Arreglo de Diferencias Relativas a Inversiones (en adelante "CIADI»). Sobre este punto, es necesaria una aclaración inicial: dentro de la variedad de mecanismos sobre arbitraje de inversiones existentes, hemos escogido al CIADI porque, cuantitativamente, es el principal sistema utilizado para dirimir esta clase de reclamaciones y, en consecuencia,

2 Esta noción de autonomía responde a la idea de que en el sistema de justicia internacional, cada tribunal existe de forma independiente respecto de los restantes, sin que se identifiquen relaciones jerárquicas u otras formas institucionales de relación y coordinación entre éstos. Una buena explicación de lo anterior fue expuesta en el famoso caso Tadic, en donde el Tribunal Penal para la Ex Yugoslavia nítidamente sentenció: "El derecho internacional, debido a que carece de una estructura centralizada, no contempla un sistema judicial integrado que opere sobre la base de una división ordenada de trabajo entre varios tribunales, en donde ciertos aspectos o componentes de la jurisdicción puedan ser centralizados o conferidos a uno de ellos, pero no a los demás. En el derecho internacional, cada tribunal constituye un sistema autónomo (a menos que exista disposición en contrario)". Prosecutor v. Tadic, Decision on the Defence and Motion for Interlocutory Appeal on Jurisdiction, Case No. IT-94-1, ICTY Appeals Chamber (1995), para. 11. Sin embargo, la autonomía puede abordarse no sólo desde el referido punto de vista funcional, sino también considerando el tipo de normas que dichos tribunales y cortes aplican. Sobre este último punto, una buena explicación se encuentra contenida en el Reporte de la Comisión de Derecho Internacional de las Naciones Unidas Fragmentation of International Law: difficulties arising from the diversification and expansion of International Law, finalizado por Martii KosKenNiemI, A/CN.4/L.682, 2006, pp. 65-99, para. 123-194. 
existe un cúmulo suficiente de jurisprudencia útil para trazar una panorámica que ilustre adecuadamente el objeto de este artículo ${ }^{3}$.

Como podría razonablemente aventurarse, se trata de una temática extensa que podría dar lugar a infinitos ejercicios de comparación. Por ello, nos centraremos en el estudio de la forma en que los tribunales arbitrales del CIADI han considerado la jurisprudencia de la CIJ al conocer de algunas materias específicas, sustantivas y procedimentales: el estado de necesidad, la cláusula de «la nación más favorecida», los actos unilaterales del Estado y las medidas provisionales. Para ello, en la primera parte nos referiremos de manera general al valor que detentan las decisiones previas de tribunales y cortes internacionales en tanto fuente subsidiaria de determinación de las reglas de derecho, con especial mención a lo establecido por la CIJ y por tribunales arbitrales del CIADI. En un posterior nivel de análisis, se intentará desentrañar el grado de influencia específica de la jurisprudencia de la CIJ en casos específicos conducidos ante el CIADI, en las cuatro temáticas antes mencionadas. Al final, algunas conclusiones serán propuestas.

\section{Inexistencia del precedente}

A lo primero que conviene referirse es al valor que en general tienen las decisiones judiciales en la práctica de la justicia internacional. En estricto rigor, el derecho internacional no reconoce formalmente la regla del precedente. Normativamente, el artículo 59 del estatuto de la CIJ consagra explícitamente esta afirmación, al disponer que: "La decisión de la Corte no es obligatoria sino para las partes en litigio y respecto del caso que ha sido decidido". Coherente con ello, el artículo 38 del mismo instrumento -disposición que identifica a las fuentes del derecho internacional- estipula que las decisiones judiciales sólo constituyen un "medio auxiliar para la determinación de las reglas de derecho".

Similar principio se repite tratándose del arbitraje de inversiones: el artículo 53(1) del Convenio sobre arreglo de diferencias relativas a inversiones entre Estados y nacionales de otros Estados de 18 de marzo de 1965 (en adelante "Convenio de Washington») se refiere al efecto del laudo que ha de pronunciar un tribunal arbitral constituido bajo las reglas del CIADI, señalando que dicha resolución será “obligatoria para las partes” que se sometieron al respectivo procedimiento ${ }^{4}$.

3 De acuerdo con las estadísticas del CIADI al 31 de diciembre de 2013, el Centro ha registrado un total de 407 casos de arbitraje bajo el Convenio de Washington, y 43 al amparo del Reglamento del Mecanismo Complementario. Fuente: Carga de Casos del CIADI-Estadísticas (Edición 2014-1), p. 8. El documento es publicado por el Secretariado del CIADI, y se encuentra disponible electrónicamente en: https://icsid. worldbank.org/ICSID/FrontServlet?requestType=ICSIDDocRH\&actionVal=CaseLoadStatistics [consulta: 15 de mayo de 2014].

4 Diversas normas que consagran el efecto relativo de la sentencia se repiten en los marcos normativos de otros 
Este efecto relativo de las sentencias ha sido confirmado jurisprudencialmente tanto por la CIJ como por distintos tribunales arbitrales del CIADI, desde un doble de vista, sosteniendo que no se encuentran obligados ni por sus propios fallos anteriores, ni por las resoluciones emitidas por otros órganos judiciales pertenecientes al sistema internacional (esto es, por precedentes externos).

Así, la Corte Permanente de Justicia Internacional (en adelante "CPJI»), refiriéndose al artículo 59 de su Estatuto, tempranamente afirmó que: "El propósito de este artículo es simplemente evitar que principios jurídicos aceptados por la Corte en un caso particular resulten obligatorios para otros Estados o en otros casos" ${ }^{\text {" }}$. Posteriormente, la $\mathrm{CIJ}$ adoptó similar fórmula para referirse a que la sentencia sólo tiene efecto obligatorio respecto de las partes que se someten al procedimiento. En el caso sobre la Plataforma continental entre Libia y Malta, hizo referencia al mencionado dictamen de la CPJI, repitiendo idéntico razonamiento y concluyendo que los derechos de Italia, tercer Estado que solicitaba intervenir en la disputa en cuestión, se encontraban adecuadamente salvaguardados justamente en virtud del referido efecto relativo de la sentencia consagrado en el artículo 59 del Estatuto ${ }^{6}$. A través de estas interpretaciones, la CIJ ha dejado de manifiesto que cada caso específico debe ser resuelto conforme su propio mérito y de acuerdo con sus particulares circunstancias, y que la motivación y obligaciones de un caso difícilmente pueden ser extrapoladas a otro asunto sin un detallado razonamiento que lo justifique 7 .

Desde el otro lado del espectro, varios tribunales arbitrales del CIADI han dado eficacia al contenido del artículo 53(1) del Convenio de Washington. Así, en Enron v. Argentina, el panel arbitral dictaminó: "El Tribunal concuerda con la visión expresada por la República Argentina en la audiencia sobre jurisdicción llevada a cabo en esta disputa, respecto al efecto de que la decisiones de tribunales del CIADI no constituyen precedentes obligatorios y que cada caso debe ser examinado a la luz de sus propias circunstancias" ${ }^{\prime}$. En AES Corp. v. Argentina -presumiblemente el caso del CIADI en el

órganos judiciales internacionales. Por ejemplo, el artículo 33(2) del Estatuto del Tribunal Internacional de Derecho del Mar dispone que: "El fallo sólo tendrá fuerza obligatoria para las partes respecto de la controversia que haya sido decidida". La misma idea se reitera en el artículo 37 del Estatuto de la Corte Centroamericana de Justicia.

5 Certain German Interests in Polish Upper Silesia (Germany v. Poland), Merits, Judgment, PCIJ, Series A, No 7, 1926, p. 19.

6 Continental Shelf(Libyan v. Malta), Application to Intervene, Judgment, I.C.J. Reports 1984, para. 42.

7 JaCOB, Marc. "La Función Sistémica del Precedente: Perspectivas del Derecho Internacional". En: Ferrer Mac-Gregor, Eduardo et al (eds.). La Justicia Constitucional y su Internacionalización. ¿Hacia un Ius Constitucionale Commune en América Latina?, Tomo II. Instituto de Investigaciones Jurídicas, Universidad Autónoma de México, Ciudad de México. 2010, p. 694.

8 Enron Corporation y Pondeorsa Assets, L.P. v. Argentina, Caso CIADI No ARB/01/3, Decisión sobre jurisdicción (demanda adicional) (02.08.2004), para. 25. 
cual mayor discusión se produjo respecto del valor jurídico de las decisiones previas-, el tribunal arbitral fue meridianamente claro en cuanto al tratamiento que de acuerdo con el derecho internacional público en general, y con el Convenio de Washington en particular, debía otorgársele a las decisiones previas, señalando que: "Cada decisión o laudo emitido por un Tribunal del CIADI sólo es obligatorio respecto de las partes de la disputa resuelta a través de dicha decisión o laudo. No existe hasta ahora regla del precedente en el derecho internacional general"'.

2. ... pero

Es también efectivo que con bastante frecuencia las decisiones judiciales son consideradas por los propios tribunales como evidencia del derecho, intentando salvaguardar adecuados niveles de consistencia y coherencia en la interpretación y aplicación de similares reglas y principios ${ }^{10}$. De esta forma, los órganos jurisdiccionales acostumbran a otorgar valor a la predictibilidad y certeza del sistema, en el sentido de procurar que casos similares sean resueltos de manera parecida, a menos que existan poderosas razones que justifiquen un proceder diferente. Para identificar la idea a la que hacemos referencia, se suele utilizar la expresión "jurisprudencia constante"11.

\subsection{Consistencia interna}

Esta búsqueda de predictibilidad es sencilla de identificar en la práctica de la CIJ, si analizamos las numerosas ocasiones en las cuales ha hecho mención en forma de referencias o extractos a su propia jurisprudencia para justificar un razonamiento, sin efectuar distinciones de si se trata de decisiones pronunciadas en asuntos contenciosos o derivados de su práctica consultiva. Y lo ha efectuado desde los comienzos de su actividad. Recién establecida, la Corte dejó en claro el valor del trabajo de su antecesora, al hacer suyas en el caso del Canal de Corfú "las visiones expresadas por la Corte Permanente de Justicia Internacional con respecto a cuestiones similares de interpretación”12. De igual modo, en la opinión consultiva sobre Reparación por daños sufridos al servicio de las Naciones Unidas,

9 AES Corporation v. Argentina, ICSID Case No. ARB/02/17, Decision on Jurisdiction (26.04.05), para. 23 (d).

10 Crawford, James. Brownlie's Principles of International Law. Oxford University Press, Oxford, 2008, pp. 37-38.

$11 \mathrm{Al}$ respecto, Guillaume, Gilbert. "The Use of Precedents by International Judges and Arbitrators”. Journal of International Dispute Settlement, Vol. 2, no 1, 2011, pp. 5-23; Pellet, Alain. "The Case Law of the ICJ in Investment Arbitration” (Lalive Lecture). ICSID Review, Vol. 28, no 2, 2013, pp. 223-240 y Schreuer, Christoph. "Diversity and Harmonization of Treaty Interpretation in Investment Arbitration". Transnational Dispute Management, Vol. 3, n 2, 2006, pp. 1-24.

12 Corfu Channel (United Kingdom v. Albania), Judgment, I.C.J. Reports 1949, p. 24. 
la CIJ teorizó sobre la capacidad de las Naciones Unidas para presentar una demanda internacional en contra de un Gobierno determinado, con el ánimo de obtener reparaciones respecto de ciertos daños causados por éste. En su decisión, recordó el denominado principio de eficacia, conforme al cual bajo el derecho internacional debe estimarse que una organización internacional (en este caso, la ONU) cuenta con aquellos poderes que resulten esenciales para el adecuado cumplimiento de sus responsabilidades, aún cuando éstos no estuvieren expresamente previstos en la $\mathrm{Carta}^{13}$. Esto fue una confirmación de lo establecido por la CPJI en la opinión consultiva sobre Ciertas atribuciones regulatorias de la Organización Internacional del Trabajo ${ }^{14}$.

En casos más recientes destaca la opinión consultiva sobre la Construcción de un muro en territorio ocupado de Palestina, oportunidad en la cual la CIJ estimó que, entre las varias obligaciones violadas por Israel, se incluían algunas del tipo erga omnes. En la calificación de esta clase de obligaciones, recordó la primera mención que de ellas efectúo años antes en el famoso caso Barcelona Traction, reiterando el criterio definitorio establecido en aquél entonces ${ }^{15}$. En la misma opinión consultiva, la CIJ trajo a colación las variadas oportunidades en las cuales se había visto en la necesidad de realizar una interpretación amplia (o derechamente una reformulación) de la pregunta específica sometida a su conocimiento, debido a su falta de precisión. Para respaldar lo adecuado de dicho proceder, efectuó un extenso listado en el que incluyó una serie de otros casos en los cuales actuó de similar modo ${ }^{16}$.

Finalmente, en el reciente fallo sobre la Delimitación marítima entre Perú y Chile, la Corte también hizo mención en varios acápites a su propia práctica. Una de las ocasiones más relevantes fue cuando reiteró el criterio sostenido en el caso de la Disputa territorial y marítima entre Nicaragua y Honduras, señalando que al ser el establecimiento de un límite marítimo una materia de "significativa importancia", las evidencias recogidas para acreditar su existencia de modo tácito debían ser suficientemente convincentes y fundadas ${ }^{17}$.

De esta forma, a pesar de que no existe una obligación (deber) para la Corte de respetar

13 Reparation for Injuries Suffered in the Service of the United Nations, Advisory Opinion, I.C.J. Reports 1949, pp. 182-183.

14 Competence of the ILO to Regulate, Incidentally, the Personal Work of the Employer, Advisory Opinion, PCIJ 1926, Series B, No 13, p. 18.

15 Legal Consequences of the Construction of a Wall in the Occupied Palestinian Territory, Advisory Opinion, I.C.J. Reports 2004, para. 155, refiriéndose al dictamen emitido en Barcelona Traction, Light and Power Company, Limited (Belgium v. Spain), I.C.J. Reports 1970, para. 33.

16 De hecho, en una sola parte de la opinión consultiva la Corte hizo referencia a doce casos anteriores. Vid. Legal Consequences of the Construction of a Wall, para. 38-40.

17 Maritime Dispute (Peru v. Chile), Judgment, I.C.J. General List No. 137, 27 January 2014, para. 91, citando Territorial and Maritime Dispute between Nicaragua and Honduras in the Caribbean Sea (Nicaragua v. Honduras), Judgment, I.C.J. Reports 2007 (II), para. 253. 
ni siquiera sus propios fallos, es al mismo tiempo cierto que es muy cuidadosa para evitar incurrir en flagrantes contradicciones con sus propias decisiones ${ }^{18}$. Por el contrario, más bien lo que tiende a efectuar es sumergirse en un proceso de búsqueda de razones suficientemente convincentes para decidir si se aparta de una práctica establecida, justificando sobre la base de un análisis caso a caso tales distanciamientos. Esta idea queda de manifiesto en un pasaje de la decisión sobre las objeciones preliminares presentadas por Nigeria en el caso sobre la Delimitación maritima y terrestre, que la enfrentó con Camerún. En esa oportunidad, la CIJ expresó: "Es cierto que, de acuerdo con el Artículo 59, las decisiones de la Corte obligan sólo a la partes y sólo respecto de un caso particular (...). La verdadera pregunta es si, en este caso, existe razón o no para seguir el razonamiento y conclusiones de casos anteriores"19.

La búsqueda de esta “jurisprudencia constante” también ha sido una aspiración compartida por los tribunales arbitrales que han operado en el sistema del CIADI. En todo caso, valga una importante precisión: debido a la naturaleza atomizada del sistema de solución de conflictos establecido por el Convenio de Washington, en virtud del cual diferentes tribunales arbitrales son constituidos de manera ad-hoc de las más variadas formas para resolver conflictos sobre inversiones extranjeras, existen mayores posibilidades de identificar casos de jurisprudencia divergente. Ello es así porque, por un lado, sus decisiones tienen un grado de especificidad mucho mayor que las pronunciadas por tribunales permanentes y, por otro, al hecho de que estos tribunales no deben hacerse cargo con posterioridad de decisiones pronunciadas por sus pares, pues su composición varía de caso en caso. Esta característica da cuenta de un importante contraste con lo que acontece tratándose de la $\mathrm{CIJ}^{20}$.

18 Pellet, op. cit., p. 785. Sin embargo, esto no quiere decir que la CIJ a lo largo de sus años no haya revertido decisiones previas adoptando una nueva postura en la interpretación y aplicación del derecho. En efecto, tratándose del establecimiento de criterios para demarcar fronteras marítimas, la CIJ ha dado algunas muestras de una histórica tensión existente entre el uso del método de la equidistancia, en contraposición con la búsqueda de soluciones equitativas que tomen en cuenta todas las circunstancias relevantes, configurando una jurisprudencia vacilante. Al respecto, conviene mencionar los casos North Sea Continental Shelf (Germany v. Denmark, Netherlands), I.C.J. Reports 1969; Continental Shelf (Libya v. Malta), I.C.J. Reports 1985 y Maritime Delimitation in the Area between Greenland and Jan Mayen (Denmark v. Norway), I.C.J. Reports 1993.

19 Land and Maritime Boundary between Cameroon and Nigeria (Nigeria v. Cameroon), Preliminary Objections, Judgment, I.C.J. Reports 1998, para. 28.

20 Esta idea ha sido claramente sintetizada por un tribunal arbitral constituido bajo las reglas de la UNCITRAL, en el caso Glamis Gold, Ltd. v. United States of America: "A diferencia de los órganos de adjudicación permanentes que conocen múltiples disputas (...), un panel arbitral que está centrado en una disputa particular no está confrontado con la posibilidad de que será necesario aplicar una decisión previa en un procedimiento posterior. Del mismo modo, un tribunal arbitral no se encuentra confrontado con la tarea de reconciliar sus decisiones posteriores con sus decisiones previas". Glamis Gold, Ltd. v. United States of America, Arbitral Tribunal constituted under Chapter 11 NAFTA, Award (08.06.09), para. 3. 
Sin embargo, en reiteradas oportunidades dichos tribunales arbitrales han hecho explícitas sus intenciones de salvaguardar la consistencia con decisiones anteriores. Así, en LETCO v. Liberia, el panel dio cuenta de la relación que existía entre dicha disputa y las decisiones previas de otros tribunales constituidos bajo las reglas del CIADI, señalando que: "Aunque el Tribunal no está obligado por los precedentes establecidos en otros Tribunales del CIADI, resulta no obstante instructivo considerar tales interpretaciones" 21 . Del mismo modo, en Gas Natural v. Argentina el tribunal estimó que "está satisfecho de que sus análisis y decisiones, a los que llegó en forma independiente, son compatibles con las conclusiones de otros tribunales arbitrales que debieron pronunciarse sobre cuestiones similares"22.

Por contrapartida, también es posible identificar notables casos en los cuales estos paneles han preferido subrayar la facultad que cada uno de ellos posee para adoptar sus propias decisiones en el marco del conocimiento de un caso particular, incluso si ello implica apartarse de criterios previamente establecidos. Un ejemplo de ello lo encontramos en el caso PSG v. Filipinas, en donde el respectivo panel fue enfático en marcar este contraste: "Aunque distintos tribunales constituidos bajo el sistema del CIADI debiesen en general buscar actuar consistentemente unos con otros, al final cada tribunal debe ejercer su propia competencia de acuerdo con el derecho aplicable, el cual será por definición diferente para cada TBI y cada Estado demandado. Es más, no existe doctrina del precedente en el derecho internacional, si por precedente se entiende una regla sobre el efecto obligatorio de una decisión particular. No existe jerarquía de tribunales internacionales e, incluso si la hubiera, no hay una buena razón para permitir que el primer tribunal en el tiempo resuelva asuntos para todos los tribunales posteriores"23. En un párrafo de la decisión sobre jurisdicción del ya mencionado caso Gas natural v. Argentina, el tribunal se mostró distante respecto de la obligación de considerar decisiones anteriores: "El Tribunal desea enfatizar que ha dictado su decisión de manera independiente, sin considerarse a sí mismo obligado por ningún otra decisión o laudo" 24 .

LETCO v. Liberia, ICSID Case No ARB/83/2, Award (31.03.86), para. 352.

Gas Natural SDG, S.A. v. Argentina, Caso CIADI No ARB/03/10, Decisión sobre Jurisdicción (17.06.05), para. 52.

23 SGS Société Générale de Surveillance SA v. Philippines, ICSID Case No ARB/02/6, Decision on Jurisdiction (29.01.04), para. 97.

24 Gas Natural SDG, S.A. v. Argentina, supra nota 21, para. 36. 


\subsection{Consistencia externa}

Hasta aquí hemos hecho hincapié en aquellos casos en los cuales tanto la CIJ como diferentes tribunales arbitrales del CIADI han efectuado reenvíos hacia su propia jurisprudencia, principalmente para corroborar una interpretación o aplicación del derecho existente. Pero hay notorias diferencias cuando revisamos la forma en que ambas instancias han procedido, tratándose de potenciales referencias hacia la práctica de órganos jurisdiccionales que pertenecen a otros sub-sistemas. Lo primero que llama la atención es el evidente recelo exhibido por largos ańos por la CIJ en cuanto a considerar decisiones emanadas de fuentes exógenas en sus propias resoluciones. Sólo en circunstancias excepcionales ha demostrado una tenue apertura ${ }^{25}$. Probablemente, la razón de esto sea el irresistible deseo de la Corte de resguardar desde una perspectiva simbólica su posición en cuanto principal órgano judicial de las Naciones Unidas, concibiéndose a sí misma como un órgano dotado de una mayor autoridad en cuanto a la responsabilidad por el desarrollo progresivo del derecho internacional, en comparación con otros tribunales y cortes.

Sin embargo, esta tendencia pareciera estar lentamente revirtiéndose en los últimos años, durante los cuales la Corte ha comenzado a exhibir mayores niveles de flexibilidad en las referencias a precedentes externos ${ }^{26}$. En todo caso, el común denominador de estos casos es que todos ellos han dicho relación exclusivamente con disputas interestatales, no constatándose ningún ejemplo en el cual la Corte haya efectuado una mención a resoluciones dictadas en el marco de procedimientos de arbitraje comercial internacional o de inversiones.

A su turno, en el sistema del CIADI, referencias hacia la jurisprudencia externa han sido efectuadas con mucha mayor frecuencia, sobre todo tratándose de la mención de casos sometidos al conocimiento de la CIJ. Lógicamente, esto se ha producido en un doble sentido, primero, para confirmar una determinada interpretación o, segundo, para argumentar el por qué resulta conveniente sostener la aplicación divergente de una determinada regla o principio. La mayoría de las veces, estos tribunales arbitrales suelen manifestar una notoria deferencia con los fallos de la CIJ. A modo ejemplar, en Azurix v. Argentina, el tribunal arbitral expresó: "De conformidad con el Artículo 31 de

25 La primera vez que la CIJ mencionó una decisión emanada de otro tribunal internacional fue en 1953, cuando en el caso Nottebohm reafirmó la trascendencia del principio de la competencia-competencia, a través de una mención a la serie de arbitrajes conocida como Alabama. Véase Nottebohm case (Liechtenstein v. Guatemala), Preliminary Objections, Judgment, I.C.J. Reports 1953, p. 63.

26 Por ejemplo, en Land, Island and Maritime Frontier Dispute (El Salvador v. Honduras, Nicaragua intervening), Judgment, I.C.J. Reports 1992, para. 391; en Maritime Delimitation and Territorial Questions between Qatar and Bahrain (Bahrain v. Qatar), Judgment, I.C.J. Reports 2001, para. 100, 117; y en Sovereignty over Pulau Ligitan and Pulau Sipadan (Indonesia v. Malaysia), Judgment, I.C.J. Reports 2002, para. 135; entre otros. 
la Convención de Viena, el Tribunal está obligado a considerar el significado corriente de los términos utilizados en el TBI. Las conclusiones de otros tribunales, en particular de la CIJ, deberían ayudar al Tribunal en su labor de interpretación”27.

Pero hay otras situaciones en las cuales se ha insistido en recalcar el carácter soberano y autónomo que detenta cada decisión particular, sin importar si ello implica apartarse de interpretaciones sostenidas incluso por la CIJ. En el caso Amco v. Indonesia, el Comité ad-hoc constituido para conocer el recurso de nulidad del laudo inicialmente pronunciado dictaminó: "Ni la decisión de la Corte Internacional de Justicia en el caso del Laudo del Rey de España ni la decisión del Comité ad-hoc en Klöckner son obligatorias para este Comité ad-hoc'ns.

Esta aparente disyuntiva de si persistir con un patrón establecido o realizar una interpretación diferente de ciertas reglas jurídicas, sumado a la multiplicidad de tribunales que de manera ad-hoc son conformados, ha llevado a que se ponga en duda la pertinencia de referirse a la existencia de "una" jurisprudencia del CIADI, a pesar de que algunas decisiones hacen explícita referencia a ella ${ }^{29}$. Más bien, lo que en la práctica existiría sería una suerte de jurisprudencia constante relativa a un limitado número de cuestiones jurídicas (por ejemplo, el carácter obligatorio de las medidas provisionales), y una desafortunada confusión respecto de otras (las distintas visiones sobre la definición de inversión es un perfecto ejemplo). Debido a lo anterior, "hablar de 'la' jurisprudencia del CIADI sería demasiado audaz" ${ }^{\prime 30}$.

\section{Recepción de la jurisprudencia de la CIJ en las decisiones de los tribunales arbitrales del CIADI}

Lo antes expuesto sirve como insumo para colocar en contexto varios de los aspectos que revisaremos a continuación. Las premisas iniciales que utilizaremos en este análisis son las siguientes: (a) de acuerdo con los artículos 59 del Estatuto de la CIJ y 53(1) del Convenio de Washington, las decisiones pronunciadas tanto por la Corte como por los tribunales arbitrales del CIADI sólo tienen efecto obligatorio para la resolución

\footnotetext{
27 Azurix Corp. v. Argentina, Caso CIADI No ARB/01/12/, Laudo (14.07.06), para. 391.

28 Amco Asia Corporation and others v. Indonesia, ICSID Case No ARB/81/1, Decision on Annulment (16.05.86), para. 44.

29 Mr. Franck Charles Arif v. Republic of Moldova, ICSID Case No ARB/11/23, Award (08.04.13), para. 383 ("Más aún, bajo la jurisprudencia del CIADI [...]”), y para. 630 ("Existen dos estándares en la jurisprudencia del CIADI en relación con los costos [...]").

30 Pellet, op. cit., pp. 223-25.
} 
de la disputa específica en la cual se dictan; (b) ambas normas han sido expresamente mencionadas en la resolución de una serie de controversias, enfatizando el efecto relativo de las sentencias; (c) lo anterior no obsta a que, en forma recurrentemente, tanto la CIJ como los paneles del CIADI efectúen referencias a casos en los cuales se han discutido cuestiones similares; (d) la CIJ ha mostrado una escasa apertura mencionar en sus fallos la jurisprudencia emanada de otros tribunales y cortes internacionales, pero, por contrapartida, manifiesta una marcada tendencia a apoyar sus razonamientos en sus propios precedentes; (e) tratándose del CIADI, sus tribunales arbitrales son proclives a referirse tanto a procedentes "internos" como "externos", sea para coincidir, sea para adoptar nuevas interpretaciones; (f) la CIJ y los paneles del CIADI en general persiguen el desarrollo de una "jurisprudencia constante", que otorgue ciertas garantías de predictibilidad a los actores del sistema.

Una de las lógicas consecuencias de la recapitulación planteada es que este estudio sólo puede efectuarse desde una perspectiva unidireccional, esto es, dando cuenta de la manera en que diferentes paneles arbitrales del CIADI han recogido la práctica de la CIJ. La relación inversa no es posible debido a la tradicional reticencia de la CIJ para referirse a la jurisprudencia de otros tribunales y cortes internacionales, a lo que se suma las escasas oportunidades en las cuales ha conocido de disputas relacionadas con inversiones ${ }^{31}$.

La última precisión tiene que ver con que los tribunales arbitrales del CIADI tienden a dar prioridad a la mención de la jurisprudencia emanada de sus propios pares (interna), más que otra clase de tribunales o cortes (externa). Este rasgo parece natural y obvio, si consideramos que son precisamente esta clase de tribunales arbitrales los encargados de conocer de modo específico esta clase de controversias, desarrollando progresivamente un tipo de jurisprudencia especializada sobre inversiones. En ocasiones, esta suerte de preferencia por los precedentes internos ha sido criticada, sobre todo cuando se analiza la valoración que los paneles efectúan de la jurisprudencia de la CIJ. En este sentido, Pellet afirma que uno de los elementos más confusos es la extrema diversidad en cuanto a la intensidad con la cual los tribunales del CIADI hacen referencia a los fallos de la CIJ. Esto se traduce en que recurrentemente los paneles se sitúen frente a la práctica de la CIJ en una actitud sumamente vacilante, que va desde la absoluta indiferencia por sus decisiones, hasta considerarlas muchas veces como fuente de una autoridad indiscutiblemente obligatoria ${ }^{32}$.

31 La CIJ ha conocido casos que involucran inversiones extranjeras en: Certain Norwegian Loans (France v. Norway), Judgment, I.C.J. Reports 1957; Barcelona Traction, Light and Power Company, Limited (Belgium v. Spain), Order of 10 april 1961, I.C.J. Reports 1961; Elettronica Sicula S.p.A. (ELSI) (United States of America v. Italy), Judgment, I.C.J. Reports 1989; Ahmadou Sadio Diallo (Republic of Guinea v. Democratic Republic of Congo), Judgment, I.C.J. Reports 2010; y Pulp Mills on the River Uruguay (Argentina v. Uruguay), Judgment, I.C.J. Reports 2010.

32 Pellet, op. cit., p. 233. 
Esta ambivalencia se grafica adecuadamente si analizamos cómo los primeros tribunales arbitrales del CIADI evaluaron la invocación del estado de necesidad en tanto circunstancia excluyente de responsabilidad internacional por actos internacionalmente ilícitos, cuando conocieron de múltiples demandas entabladas en contra de Argentina luego del colapso político, social y económico de fines de 2001. En el primero de los casos, CMS v. Argentina ${ }^{33}$, el panel adoptó los criterios y estándares establecidos por la CIJ en su fallo relativo al caso Gabč́kovo-Nagymaros ${ }^{34}$. En efecto, no sólo compartió con la CIJ el carácter de normativa consuetudinaria que ésta otorgó al Proyecto de articulos sobre responsabilidad del Estado por hechos internacionalmente ilícitos ${ }^{35}$, sino que además recogió los requisitos acumulativos y las condiciones bajo las cuales el estado de necesidad podía ser satisfactoriamente invocado por parte de un Estado, además del estándar de prueba para ello exigido y el carácter excepcionalísimo de su acogimiento ${ }^{36}$. No obstante, el segundo de los tribunales arbitrales que conoció de una disputa similar tuvo una aproximación diametralmente opuesta respecto a la consideración de este precedente establecido por la CIJ ${ }^{37}$. En $L G \mho \mho E v$. Argentina $a^{38}$, el respectivo panel basó su argumentación exclusivamente en el Proyecto de artículos y en la normativa estipulada en el tratado bilateral de inversiones suscrito entre Argentina y Estados Unidos, sin efectuar referencia alguna a la jurisprudencia de la $\mathrm{CIJ}^{39}$. Esta llamativa omisión es sintomática de esta actitud a menudo inconsistente que venimos comentando, sobre todo si consideramos que en el campo de la regulación de la responsabilidad del Estado, la labor de la CIJ ha sido especialmente fundamental para su desarrollo progresivo, por lo que es razonable esperar una mayor invocación de sus decisiones y, presumiblemente, un mayor grado de consistencia con las mismas.

Más allá de esta materia específica, encontramos otras evidencias que demuestran mayores niveles de consideración. Si revisamos, por ejemplo, la forma en que algunos tribunales arbitrales del CIADI han abordado la invocación de la cláusula de «la

33 CMS Gas Transmission Company v. Argentina, Caso CIADI No ARB/01/8, Laudo (12.05.05).

34 Gabčikovo-Nagymaros (Hungary v. Slovakia), Judgment, I.C.J. Reports 1997.

35 Aun cuando este documento no tiene la naturaleza jurídica de tratado internacional, ha sido ampliamente validado y referido por la comunidad jurídica internacional, al afirmarse que constituye una fuente de codificación de la costumbre internacional. International LaW Commission. "Draft Articles on Responsibility of States for Internationally Wrongful Acts, with commentaries". Yearbook of the International Law Commission, Vol. II, Part Two, 2001, p. 83.

36 El extenso análisis se encuentra contenido entre los párrafos 304-394.

37 Tan opuesta fue su aproximación al análisis de la existencia de un estado de necesidad que incluso arribó a una decisión contradictoria con lo establecido en forma previa en CMS v. Argentina, acogiendo la excepción interpuesta por Argentina (aunque limitándola temporalmente).

38 LG\&E Energy Corp., LG\&E Capital Corp., LG\&E International Inc. v. Argentina, Caso CIADI No ARB/02/1, Decisión sobre Responsabilidad (03.10.06).

39 La decisión del tribunal arbitral sólo hace mención -cuando discute sobre la existencia de medidas arbitrarias y discriminatorias- al caso Elettronica Sicula SpA (ELSI) (United States of America v. Italy). 
nación más favorecida», observaremos que éstos han realizado constantes reenvíos a la jurisprudencia de la CIJ. En efecto, son tres los casos conocidos por la CIJ principalmente señalados: Compañia Petrolera Anglo-Iranito ${ }^{40}$ Derechos de los nacionales de los Estados Unidos de América en Marruecos ${ }^{41}$ y Ambatielos $^{42}$. En cuanto al CIADI, el leading case es Maffezini ${ }^{43}$. En este último, el panel efectuó un largo análisis a la luz de los tres casos resueltos por la CIJ, específicamente para la determinación de la pertinencia de la aplicación de la cláusula de la nación más favorecida en la disputa en cuestión. Para ello, cuando discutió sobre cuál debía ser el trato básico que rige los derechos del beneficiario de la cláusula, se refirió al caso de la Petrolera Anglo-Iraní4 . Más adelante, al abordar el problema de si podía considerarse que las disposiciones sobre solución de controversias contenidas en un tratado con un tercero están razonablemente vinculadas con el tratamiento justo y equitativo al que se aplica la cláusula de la nación más favorecida en los tratados básicos sobre comercio, navegación o inversiones, recurrió al dictamen pronunciado por la CIJ en el caso Derechos de los nacionales de los Estados Unidos en Marruecos ${ }^{45}$. Por último, el tribunal arbitral mencionó Ambatielos para ilustrar situaciones en las cuales un ciudadano extranjero no es tratado por los tribunales de otro Estado de acuerdo con los estándares a los cuales se encuentran sometidos otros extranjeros que gozaban del tratamiento de la nación más favorecida de acuerdo con los tratados vigentes ${ }^{46}$. Más adelante, en Siemens $v$. Argentina ${ }^{47}$, el tribunal arbitral repitió similar patrón de conducta, aunque profundizó en las referencias hacia los tres casos conducidos por la $\mathrm{CIJ}^{48}$. Pero como ya existía un antecedente en el propio sistema del CIADI, el panel descansó en gran parte de sus argumentaciones en Maffezini, con miras a alcanzar conclusiones consistentes ${ }^{49}$.

Sin embargo, la decisión posterior pronunciada en Salini v. Jordania ${ }^{50}$ se planteó desde una perspectiva significativamente diferente al momento de valorar los precedentes de la CIJ en relación con los alcances de la cláusula de la nación más favorecida. En la

40 Anglo-Iranian Oil Co. (United Kingdom v. Iran), Judgment (Jurisdiction), I.C.J. Reports 1952.

41 Rights of Nationals of the United States of America in Morocco (France v. United States), Judgment, I.C.J. Reports 1952.

42 Ambatielos (merits: obligation to arbitrate) (Greece v. United Kingdom), Judgment, I.C.J. Reports 1953.

43 Emilio Agustín Maffezini v. Reino de España, Caso CIADI No ARB/97/7, Decisión sobre excepciones a la jurisdicción (25.01.00).

44 Maffezini v. España, para. 44.

45 Maffezini v. España, para 47.

46 Maffezini v. España, para. 48.

47 Siemens A.G. v. República Argentina, Caso CIADI No ARB/02/8, Decisión sobre Jurisdicción (03.08.04).

48 Siemens A.G. v. Argentina, para. 94-102 (en lo relativo al razonamiento efectuado por el tribunal arbitral).

49 Argentina realizó permanentes referencias a Maffezini, las que fueron rebatidas por la demandante. El tribunal arbitral, por su parte, coincidió en varios de los puntos jurídicos discutidos con el razonamiento de este penal anterior.

50 Salini Costruttori S.p.A. and Italstrade S.p.A. v. Kingdom of Jordan, ICSID Case No ARB/02/13, Decision on Jurisdiction (29.11.04). 
discusión sobre si la cláusula podía o no ser invocada para los propósitos de la solución de controversias, el tribunal arbitral descartó la pertinencia de aplicar los mismos razonamientos para el caso en comento contenidos en las decisiones de la CIJ en Petrolera Anglo-Iraní y Derechos de los nacionales ${ }^{5}$. En relación con Ambatielos, los miembros del panel sostuvieron que al no haberse pronunciado el tribunal sobre los méritos, su extrapolación resultaba dificultosa. Un hecho que llama la atención es la mención efectuada por el tribunal arbitral a las opiniones disidentes de los jueces que apoyaron el planteamiento formulado en su oportunidad por Grecia, replicando la premisa de que "la cláusula no puede ser extendida hacia materias distintas de aquellas respecto de las cuales ha sido estipulada" (comercio y navegación) y que por consiguiente "no puede ser aplicada a la administración de justicia" ${ }^{\prime 2}$.

A modo de contraste, es también destacable la mención a un reciente fallo en el que también se discutió latamente sobre si la cláusula de la nación más favorecida era aplicable a las disposiciones sobre solución de controversias, en el marco de un procedimiento conducido ante la Corte Permanente de Arbitraje. En ICS Inspection v. Argentina ${ }^{53}$, refiriéndose a los mencionados tres casos de la CIJ, el tribunal arbitral, aunque arribó a conclusiones parcialmente diversas, mostró una especial deferencia, señalando claramente que "estos casos deben tenerse en cuenta no sólo como jurisprudencia relativa a la interpretación correcta de las cláusulas de NMF, sino asimismo como precedentes que influyeron en la redacción de tratados posteriores" ${ }^{\prime 4}$.

En otros asuntos, los paneles han expresado un apoyo incondicional respecto de conclusiones contenidas en sentencias de la CIJ, haciendo suyos importantes planteamientos que han venido a dilucidar algunos puntos jurídicos hasta entonces arduamente discutidos en doctrina y jurisprudencia. En algunos casos, esto se ha efectuado desafortunadamente sin profundizar en las reflexiones. Hasta hace algunos años, era común identificar distintas interpretaciones respecto de la obligatoriedad del cumplimiento de las medidas

\footnotetext{
Salini v. Jordan, para. 106.

Salini v. Jordan, para. 106.

ICS Inspection and Control Services Limited v. República Argentina, Caso CPA o 2010-9, Laudo sobre Jurisdicción (10.02.12).

54 ICS Inspection v. Argentina, para. 291. Aunque sin referirse explícitamente a sentencias pronunciadas por la CIJ, el panel del CIADI constituido en Impregilo v. Argentina también compartió la extensión de la cláusula de la NMF al régimen de solución de controversias, señalando que: "El Tribunal Arbitral es de la opinión que el término 'tratamiento' es en sí mismo suficientemente amplio como para ser también aplicable a asuntos procedimentales como la solución de disputas", añadiendo que: "El Tribunal Arbitral nota además que existe un volumen masivo de jurisprudencia que indica que, al menos cuando hay una cláusula de la NMF aplicable a 'todas las materias' reguladas en el TBI, las cláusulas sobre solución de controversias más favorables de otros TBIs serán incorporadas. Casos relevantes son Maffezini, Gas Natural, Suez, Suez and Camuzzi. Impregilo S.p.A. v. República Argentina, Caso CIADI No ARB/07/17, Laudo (21.06.2011), para. 99 y 104 respectivamente.
} 
provisionales dispuestas por un tribunal o corte internacional, pendiente la decisión sobre los méritos. En parte, esta disparidad se debía a la redacción de los respectivos instrumentos que confieren esta facultad, específicamente en lo relativo a la diferencia producida entre los verbos "indicar" 55 , "recomendar" 56 y "ordenar" 57 . Con anterioridad al caso LaGrand, la CIJ formuló sólo tangenciales planteamientos, sin tomar postura definitiva sobre la obligación de cumplimiento de esta clase de medidas cautelares, sino que más bien limitándose a desaprobar su incumplimiento ${ }^{58}$. La afirmación más clara, aunque no del todo concluyente, la pronunció en el caso Nicaragua, en donde señaló que: "Cuando la Corte concluye que la situación requiere que medidas de este tipo deben ser adoptadas, incumbe a cada una de las partes tomar seriamente en cuenta las indicaciones de la Corte"

$\mathrm{Al}$ menos en lo que a la jurisprudencia de la CIJ se refiere, la discusión parece haberse resuelto luego del caso LaGrand. Poniendo punto final al debate, la CIJ explícitamente concluyó: "Se deriva del objeto y propósito del Estatuto, así como de los términos del Artículo 41 cuando se leen en su contexto, que la facultad para indicar medidas provisionales supone que tales medidas deben ser obligatorias, en la medida en que la facultad en cuestión se basa en la necesidad, cuando las circunstancias lo requieren, de salvaguardar y evitar un perjuicio a los derechos de las partes" ${ }^{\prime 60}$.

En el arbitraje sobre inversiones, antes de LaGrand, algunos tribunales del CIADI actuaron con una lógica parecida a la sostenida por la CIJ, haciendo mención a las consecuencias derivadas del desacato a tales medidas, más que a la obligatoriedad de su cumplimiento. Por ejemplo, en MINE v. Guinea, el panel manifestó que la ausencia de cumplimento por parte de una de las partes de las recomendaciones sobre medidas provisionales sería considerada al momento de decidir sobre los méritos de la disputa ${ }^{61}$.

55 El artículo 41(1) del Estatuto de la Corte Internacional de Justicia señala que: "La Corte tendrá la facultad para indicar, si considera que las circunstancias así lo exigen, las medidas provisionales que deban tomarse para resguardar los derechos de cada una de las partes".

56 El artículo 47 del Convenio de Washington, que establece el CIADI, dispone que: "Salvo acuerdo en contrario de las partes, el Tribunal, si considera que las circunstancias así lo requieren, podrá recomendar la adopción de aquellas medidas provisionales que considere necesarias para salvaguardar los respectivos derechos de las partes".

57 El Estatuto del Tribunal de Derecho del Mar, en su artículo 25(1) menciona: "De acuerdo con el artículo 290, el Tribunal y su Sala de Controversias sobre Fondos Marinos estarán facultados para ordenar medidas provisionales".

58 Por ejemplo, en Nuclear Tests (Australia v. France), Judgment, I.C.J. Reports 1974, para. 19; Nuclear Tests (New Zealand v. France), Judgment, I.C.J. Reports 1974, para. 19 y United States Diplomatic and Consular Staff in Tehran (United States v. Iran), Judgment, I.C.J. Reports 1980, para. 75, 93.

59 Military and Paramilitary Activities in and against Nicaragua (Nicaragua v. United States), Judgment, I.C.J. Reports 1986, para. 289.

60 LaGrand (Germany v. United States), Judgment, I.C.J. Reports 2001, para. 102.

61 Maritime International Nominees Establishment (MINE) v. Republic of Guinea, ICSID Case No ARB/84/4, 
Una aproximación un poco más cercana al planteamiento de la CIJ en LaGrand se adoptó en Maffezini, aunque sin mencionar la jurisprudencia de esta última ${ }^{62}$.

La recepción íntegra por el CIADI del precedente establecido por la CIJ en LaGrand finalmente se produjo sólo unos pocos meses después. En Pey Casado v. Chile, el tribunal arbitral recogió en su totalidad la clarificación efectuada por la CIJ, concluyendo que: "Este asunto, que ha suscitado tanta polémica en la doctrina, puede decirse que ya ha sido resuelto a la luz de la jurisprudencia y, como hemos de ver más adelante, a la luz de una sentencia recientemente dictada por la Corte Internacional de Justicia" ${ }^{63}$. En consecuencia, el asunto parece haberse despejado, dando paso a una práctica constante que reconoce su carácter obligatorio, reiterada en Tokios Tokelés v. Ucrania ${ }^{64}$, Occidental Petroleum v. Ecuador ${ }^{65}$, y City Oriente v. Ecuador ${ }^{66}$, entre otros ${ }^{67}$.

Por último, los actos unilaterales han sido otro aspecto substantivo en el cual la jurisprudencia de la CIJ ha tenido impacto en la labor de los tribunales arbitrales del CIADI. En Ceskoslovenska Obchodni Banka, A.S. v. Eslovaquia ${ }^{68}$, el razonamiento del panel estuvo notoriamente influenciado por cuatro dictámenes de la CIJ. El demandante checo invocó como base de la jurisdicción del CIADI, entre otros instrumentos, una publicación en el diario oficial efectuada por una alta autoridad eslovaca, por medio de la cual se anunciaba que el respectivo tratado bilateral de inversiones (República Checa y Eslovaquia) ya se encontraba en vigor. En su opinión, este hecho, unido a la notifi-

Award (06.01.88), 4 ICSID Reports 69. Específicamente, el tribunal arbitral indicó que: "Tomando en cuenta la limitación del Art. 47, en cuanto a que el tribunal meramente 'recomienda' más que 'ordena', las medidas provisionales dispusieron que el tribunal tomaría en cuenta en su laudo los efectos del incumplimiento por MINE de sus recomendaciones".

62 Emilio Agustín Maffezini v. Reino de España, ICSID Case No ARB/97/7, Resolución Procesal No. 2 (28.10.99), para. 9: "La autoridad del Tribunal para decidir sobre la adopción de medidas provisionales no es menos obligatoria que la de un laudo arbitral definitivo. Por consiguiente, para los efectos de la presente Resolución Procesal, el Tribunal estima que la palabra 'recomendar' tiene un valor equivalente a la palabra 'dictar"'.

63 Victor Pey Casado y Fundación Presidente Allende v. República de Chile, Caso CIADI No ARB/98/2, Decisión sobre Medidas Provisionales (25.09.01), para. 17.

64 Tokios Tokelés v. Ukraine, ICSID Case No ARB/02/18, Order No 1, Provisional Measures (01.07.03), para. 4.

65 Occidental Petroleum Corporation and Occidental Exploration and Production Company v. Ecuador, ICSID Case No ARB/06/11, Decision on Provisional Measures (17.08.07), para. 58.

66 City Oriente Limited v. Ecuador and Empresa Estatal Petróleos del Ecuador, ICSID Case No ARB/06/21, Decision on Provisional Measures (19.11.07), para. 92.

67 Un notable distanciamiento de esta consistente práctica lo encontramos en Caratube International Oil Company LLP v. Republic of Kazakhstan, ICSID Case No ARB/08/12, Decision on Provisional Measures (31.07.09), para. 67. En este caso, el panel afirmó respecto de la obligación de cumplimiento de las medidas provisionales: "En este contexto debe notarse que, de acuerdo a la Regla 39, el Tribunal no puede ordenar, sino sólo puede recomendar medidas provisionales en procedimientos ante el CIADI".

68 Ceskoslovenska Obchodni Banka, A.S. v. Eslovaquia, ICSID Case No ARB/87/4, Decision on Objections to Jurisdiction (24.05.1999). 
cación de la solicitud de arbitraje, bastaban para confirmar la jurisdicción del CIADI. No obstante, la complicación radicó en que poco después de la publicación original, el Gobierno de Eslovaquia publicó una nota correctiva, declarando al tratado bilateral de inversiones como inválido.

La discusión entonces consistió en si era o no posible considerar que la publicación original en el diario oficial efectuada por Eslovaquia constituía un acto unilateral que otorgaba fundamento suficiente para la aplicación del tratado bilateral en cuestión, el que a su turno contenía la cláusula que otorgaba jurisdicción del CIADI. En su deliberación, el tribunal arbitral citó un pasaje de los dos casos $N u c l e a r ~ T e s t s^{69}$, reiterado en Nicaragua", conforme al cual "la intención del Estado que formula la declaración debe ser obligatoria de acuerdo con sus términos, de acuerdo con los principios de derecho internacional aplicables a las declaraciones unilaterales" ${ }^{\prime 1}$. En sintonía con lo resuelto por la CIJ en el caso sobre la Plataforma Continental del Mar del Norte, el tribunal arbitral estimó que estos principios no deben ser "ligeramente presumidos", y que además requieren "un curso de conducta muy consistente" 72 . Basándose entonces en estos parámetros de apreciación establecidos por la CIJ, el tribunal arbitral concluyó que la intención de Eslovaquia en cuanto a obligarse mediante la declaración original publicada en el diario oficial no podía ser establecida.

De mayor trascendencia para la práctica de futuros tribunales arbitrales, debido a su potencial aplicación general, parece ser lo recientemente resuelto en Tidewater $v$. Venezuela, en donde se discutió sobre la interpretación y posterior alcance que debía darse al artículo 22 de la Ley de Promoción y Protección de Inversiones de Venezuela, respecto del cual los demandantes sostenían que constituía un consentimiento abierto al arbitraje del CIADI. El tribunal arbitral manifestó que efectivamente la Ley de Inversiones era un acto unilateral efectuado en el marco de un tratado internacional que reconoce dicha posibilidad. En ese sentido, consideró dicho acto unilateral como análogo a una declaración unilateral formulada por un Estado que acepta la jurisdicción obligatoria de la CIJ en el contexto del artículo 36(2) de su Estatuto, y por lo tanto, procedió a analizar la práctica de dicha Corte al respecto ${ }^{73}$. En el asunto de las Pesquerías,

69 Nuclear Tests (Australia v. France), supra nota 57, para. 43; Nuclear Tests (New Zealand v. France), supra nota 57, para. 46.

70 Military and Paramilitary Activities in and against Nicaragua (Nicaragua v. United States), Jurisdiction and Admissibility, I.C.J. Reports 1984, para. 59.

71 Ceskoslovenska Obchodni Banka, A.S. v. Eslovaquia, supra nota 67, para. 46.

72 North Sea Continental Shelf(Germany v. Denmark/Netherlands), Judgment, I.C.J. Reports 1969, para. 28.

73 El tribunal arbitral sostuvo que: "una declaración unilateral que presuntamente constituye consentimiento a la competencia de un tribunal internacional debe interpretarse conforme al planteamiento enunciado por la Corte Internacional de Justicia al interpretar declaraciones de aceptación de la competencia de la Corte". Tidewater Inc. et. al v. República Bolivariana de Venezuela, Caso CIADI No ARB/10/5, Decisión sobre la 
la CIJ consideró que las declaraciones unilaterales de aceptación de su jurisdicción debían ser interpretadas en base a un método propio (al que calificó como sui generis), diferente al contemplado en la Convención de Viena sobre Derecho de los Tratados ${ }^{74}$, cuya principal característica es que debe realizarse en forma compatible con el efecto que pretende lograr el propio Estado. A la luz a lo dispuesto en esta decisión de la CIJ, sumada a la pronunciada antes en el caso de la Compañía Petrolera Anglo-Irani ${ }^{75}$, el panel concluyó que el artículo 22 de la Ley de Inversiones debía ser interpretado de buena fe, en sus propios términos, teniendo en cuenta las palabras utilizadas, en forma natural y razonable, y considerando la intención del Estado, la que se deduce no sólo del texto sino también del contexto, circunstancias y fines que se trata de alcanzar ${ }^{76}$.

\section{Conclusiones}

A través de la revisión de diferentes asuntos jurídicos, este artículo ha exhibido una panorámica que permite aproximarse al impacto de las decisiones pronunciadas por la $\mathrm{CIJ}$ en el contexto del arbitraje de inversiones del CIADI. Las temáticas analizadas han sido escogidas por estimarse las más representativas de esta influencia, pero valga la pena señalar que no se trata de un listado que se agote con el presente trabajo.

Una primera conclusión es que la inexistencia de la regla del precedente en el derecho internacional debe matizarse con la habitual práctica de los tribunales y cortes, consistente en efectuar permanentes referencias tanto a la jurisprudencia de sus pares como a la de otros órganos judiciales internacionales. En ese contexto, y a pesar de su consagrado efecto relativo, las resoluciones de la CIJ han sin duda influenciado a los paneles del CIADI, en tanto mecanismo subsidiario para la determinación y aplicación de su propio derecho. Esto no es sino una manifestación de lo dispuesto en el artículo 38(1) (d) del Estatuto de la Corte y ha acontecido tratándose de aspectos sustantivos y procedimentales.

Jurisdicción (08.02.13), para. 102(4). Referencias a la práctica de la CIJ sobre la correcta interpretación del artículo 22 de la referida Ley de Promoción y Protección de Inversiones también se encuentran en otros casos sometidos previamente al conocimiento de paneles del CIADI, destacando: Mobil Corporation, Venezuela Holdings, B.V., et al v. República Bolivariana de Venezuela, Caso CIADI No ARB/07/27, Decisión sobre Jurisdicción (10.06.2010); y CEMEX Caracas Investments B.V v. República Bolivariana de Venezuela, Caso CIADI No ARB/08/15, Decisión sobre Jurisdicción (30.12.2010).

74 La CIJ señaló: "El régimen relativo a la interpretación de declaraciones formuladas conforme al artículo 36 del Estatuto no es idéntico al establecido para la interpretación de tratados por la Convención de Viena sobre Derecho de los Tratados [...]. La Corte observa que las disposiciones de esa convención sólo pueden aplicarse por analogía en la medida compatible con el carácter sui generis de la aceptación de la competencia de la Corte". Fisheries Jurisdiction (Spain v. Canada), Judgment (Jurisdiction), I.C.J. Reports 1998, para. 46. Anglo-Iranian Oil Co., supra nota 39, p. 105.

76 Tidewater v. Venezuela, supra nota 72, para. 102(5). 
Lo segundo que puede mencionarse es que la búsqueda de consistencia entre las decisiones de diversos tribunales, o "jurisprudencia constante", ha sido una aspiración compartida por los distintos mecanismos de adjudicación internacional. Sin embargo, salvo en casos excepcionales, es positivo que esta ambición no haya importado una extrapolación irreflexiva ni automática de los postulados contenidos en prácticas anteriores, ni aun cuando encuentran su fuente en decisiones de la CIJ. Más bien, el razonamiento de los tribunales arbitrales del CIADI ha operado en un sentido inverso, esto es, transitando hacia la búsqueda de argumentos calificados que justifiquen apartarse de una jurisprudencia consolidada. En otras palabras, los dictámenes de la CIJ presumiblemente serán tenidos por ciertos por los paneles previa comprobación de la inexistencia de razones suficientemente fundamentadas que hagan conveniente un distanciamiento, conforme a las circunstancias del caso concreto.

En tercer lugar, es también efectivo que en ocasiones puntuales se constata una tendencia a dotar a la práctica de la CIJ de notables niveles de autoridad, de una especie de cuasi-obligatoriedad que operaría de facto. La razón de esto radica, probablemente, en la incuestionable ascendencia que la Corte posee respecto de los distintos regímenes de solución de controversias, fruto de su posición institucional como principal órgano judicial del sistema de las Naciones Unidas.

Finalmente, es razonable esperar que la influencia de la jurisprudencia de la CIJ se manifieste con mayor fuerza cuando los paneles del CIADI aplican normas de derecho internacional general, a menudo fundamentales para resolver disputas sobre inversiones. Ello se debe no sólo a la existencia de una práctica que con el tiempo se encuentra más consolidada, sino también al hecho de que es precisamente en esa clase de asuntos en los cuales se le reconoce a la CIJ un rol históricamente determinante, que ha contribuido decisivamente al desarrollo progresivo del derecho internacional. 
